\title{
Convergent Synthesis of Macrocycles Composed of 5-Amino-2H-1,2,4-thiadiazolin-3-one or 5-Amino-2H-1,2,4-thiadiazoline-3-thione and 1,3-Benzenedimethanethiol
}

\author{
Nam Sook Cho, ${ }^{*}$ Young Hoon Kim, and Chun Ho Lee \\ Deparment of Chemistry. Chungnam National Universitv, Daejeon 305-764, Korea \\ Received June 282004
}

Key Words : 5-Amino-2H-1,2,4-thiadiazolin-3-one, 5-Amino-2H-1,2,4-thiadiazoline-3-thione, Macrocycle, 1,3-Benzenedimethanethiol, Host molecule

Transition metal complexes with peripheral sites capable of hydrogen-bonding or $\pi$-stacking interactions to form groups have recently been used as hosts to bind neutral guests, such as aliphatic amines, ${ }^{\text {aromatic amines, }}{ }^{2}$ hydrazines. ${ }^{3}$ DNA nucleobases. ${ }^{4.5}$ amino acids. ${ }^{6.7}$ and barbiturate. ${ }^{8-18}$ This form of host is referred to as a metalloreceptor. The construction of hosts with metal ions in the scaffold has allowed the binding of many structurally sophisticated guests. Consequently, the design and study of various metalcontaining macrocycles is one of the most active and interesting areas in modern supramolecular chemistry. ${ }^{1-30}$ Coordinate covalent bond formation offers new prospects for selective molecular recognition, anion transport, and catalytic activation of electron-rich organic and inorganic substrates. It has long been recognized that multiple complementary interactions between the host and guest are of vital importance in stabilizing host-guest complexes (e.g. the chelation effect). Investigations of host-guest interactions and inclusion phenomena as well as biomimetic studies have become interesting targets. "To obtain stable, reversible adducts, there is still a clear need for neutral molecular receptors that are simple and versatile, while they are also selective. This recalls the first stages of enzymatic processes, which involve the formation of a metallo-enzyme-substrate complex. We sought to prepare macrocycles composed of 5 -membered cytosine analogues and 1.3-benzenedimethanethiol.<smiles>Nc1cc[nH]c(=O)n1</smiles><smiles>Nc1nc(=O)[nH]s1</smiles><smiles>Nc1nc(=S)[nH]s1</smiles>

Compound $\mathbf{1}^{31.32}$ is an analogue of cytosine in which the $\mathrm{C}=\mathrm{C}$ double bond moiety is replaced with a sulfur atom. In compound $2^{33}$ the carbonyl group of compound $\mathbf{1}$ is replaced with a thione. These compounds can provide sites for hydrogen bond formation and 1,3-benzenedimethanethiol can supply the chelation sites to construct complexes with metal ions. ${ }^{1-1,21,23.27}$

\section{Results and Discussion}

Compound (1) is regiospecifically $O$-alkylated under $\mathrm{NaH}$ basic conditions to give 5-amino-3-alkoxy-1,2,4-thiadiazole. Using this reaction, a macrocycle 5 , containing two 5amino-1,2,4-thiadiazole subunits linked to the 3- and 5positions of the heterocyclic unit was prepared from 1, as shown in Scheme 1. The anion of compound $\mathbf{l}$ was prepared in the presence of $\mathrm{NaH}$ in 1-methyl-2-pyrrolidinone and DMF; it was alkylated with ethylene glycol dimethanesulfonate to afford the $O$-alkylated compound (3). The formation of 3 was confirmed by its ${ }^{1} \mathrm{H}$ and ${ }^{13} \mathrm{C}$ NMR spectra. In 3. the $\mathrm{NH}$ of compound $\mathbf{1}$ was replaced by an $\mathrm{OCH}_{2} \mathrm{CH}_{2} \mathrm{OCH}_{2} \mathrm{CH}_{2} \mathrm{OMs}$ signal at 4.44, 4.38, 3.80, and $3.09 \mathrm{ppm}$ in the 'H NMR spectrum and 69.4, 69.2, 68.8, 67.8 , and $37.6 \mathrm{ppm}$ in the ${ }^{13} \mathrm{C}$ NMR spectrum. In the ${ }^{18} \mathrm{C}$ NMR, the lactam part of 1 (175.4 ppm) changed to a lactim group in $3(183.0 \mathrm{ppm})$. To provide possible chelation sites that allow the formation of complexes with metal ions, the 1,3-benzenedimethanethiol anion was produced in a 2propanol solution of sodium 2-propanoxide and alkylated with 3 to give an $S$-alkylated compound (4). The formation of 4 was also confirmed by its ' $\mathrm{H}$ and ${ }^{15} \mathrm{C}$ NMR spectra. In 4 . the $\mathrm{SH}$ of 1,3-benzenedimethanethiol was replaced by a $\mathrm{SCH}_{2} \mathrm{CH}_{2} \mathrm{OCH}_{2} \mathrm{CH}_{2} \mathrm{O}-\mathrm{I}, 2.4$-thiadiazole signal at 6.64, 4.42, $3.74,3.62$, and $2.60 \mathrm{ppm}$ in the ${ }^{\mathrm{H}} \mathrm{H}$ spectrum and 183.0 . $167.0,71.2,69.1,68.2$, and $30.9 \mathrm{ppm}$ in the ${ }^{19} \mathrm{C}$ NMR spectrum. The disappearance of the mesyl group of compound 3 at 3.09 and $37.6 \mathrm{ppm}$ in the ${ }^{1} \mathrm{H}$ and ${ }^{13} \mathrm{C}$ NMR spectra. respectively and the appearance of a 1,3-xylenyl group at 7.27-7.17 and $3.74 \mathrm{ppm}$ in the ' $\mathrm{H}$ spectrum and $138.6,129.6$. $128.6,127.6$, and $36.7 \mathrm{ppm}$ in the ${ }^{15} \mathrm{C}$ NMR spectrum also supported the formation of 4 . The target macrocycle was obtained wa cyclization involving $N, N$-diacylation of 4 at the $\mathrm{NH}_{2}$ of the 1,2,4-thiadiazole rings using diglycolyl chloride with a high dilution technique. The diglycolyl chloride solution was added to $\mathrm{CH}_{2} \mathrm{Cl}_{2}$ solution of 4 over a $24 \mathrm{hr}$ period. The structure of the macrocycle was firmly established by ${ }^{\prime} \mathrm{H}$ and ${ }^{1} \mathrm{C}$ NMR. IR, and HRMS. The successful macrocyclization of 4 to 5 was supported by evidence of $N$ acylation, indicated by the $\mathrm{NHCOCH}_{2}$ group that replaced $\mathrm{NH}_{2}$ at 12.91, and $4.51 \mathrm{ppm}$ in the ${ }^{1} \mathrm{H}$ spectrum and 167.2 
<smiles>Nc1nc(=O)[nH]s1</smiles>

1

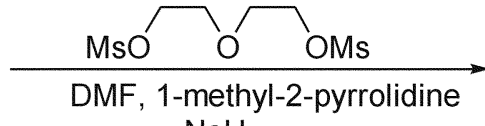

$\mathrm{NaH}$<smiles>COCCOCCOc1nsc(N)n1</smiles>
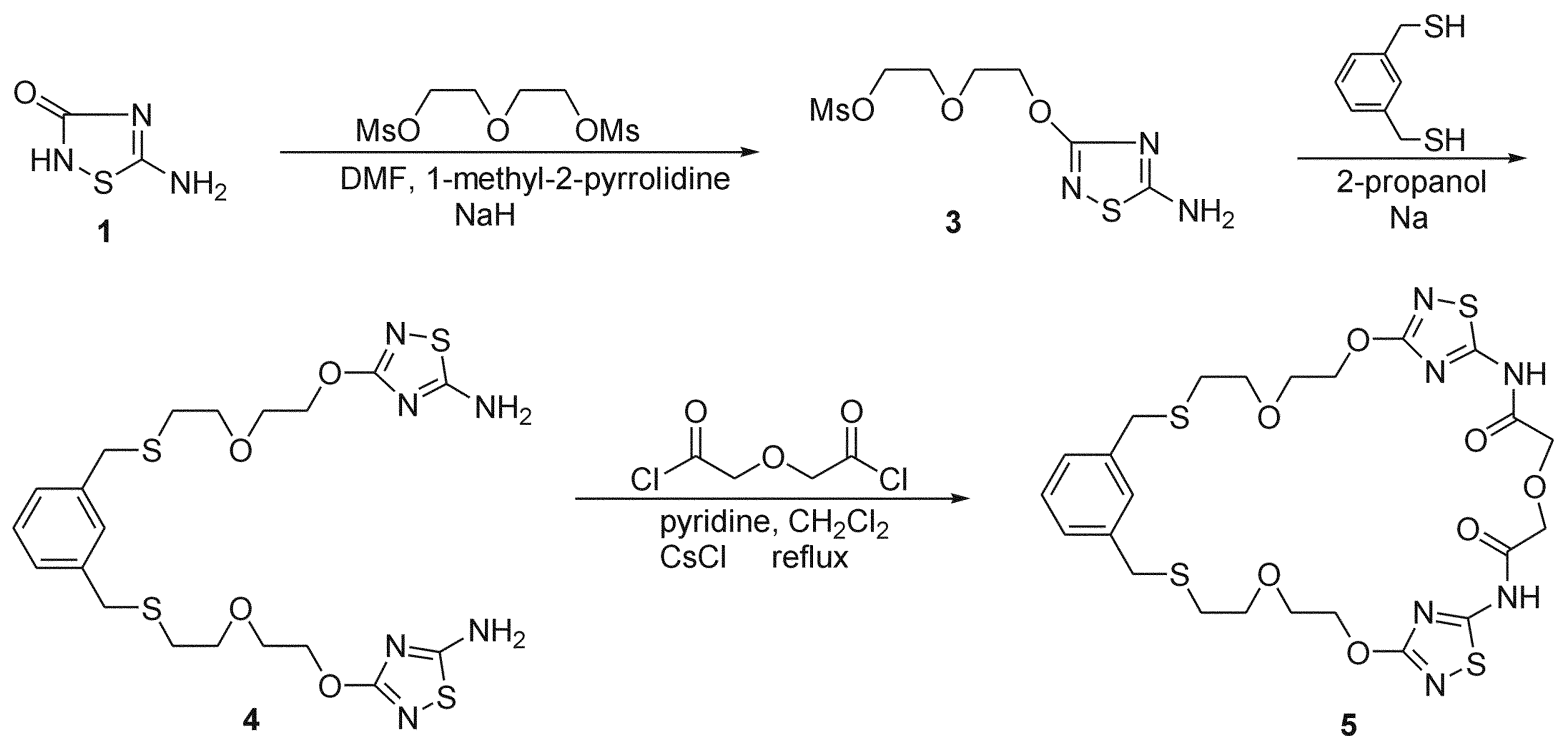

5

Scheme 1. Synthesis of macrocycle containing wo 5 -amino-2H-1,2.4-1hiadiazolin-3-one subunits.

and $71.8 \mathrm{ppm}$ in the ${ }^{1 .} \mathrm{C}$ NMR spectrum. The $\mathrm{IR}$ spectrum also shows the carbonyl group of the amide at $1701 \mathrm{~cm}^{-1}$. FAB-HRMS clearly supported the structure of 5 (Cald 643.1137. Found 643.1134).

We reported the synthesis of bis(5-amino-1,2,4-thiadiazolyl)-3,3'-disulfide (7) and the $S$-alkylation of compound (7) at the 3-position under basic conditions to afford 3alkylthio-5-amino-1,2,4-thiadiazole. ${ }^{3.3}$ Using this reaction, macrocycle 8 was synthesized, and it had the same scaffold as macrocycle 5 . The only structural difference between $\mathbf{8}$ and 5 was the atom linking the 1,2,4-thiadiazole subunit, which is sulfur in 8 and oxygen in 5 . Macrocycle 8 was synthesized as shown in Scheme 2 . The synthesis sequence of $\mathbf{8}$ differs from that of $\mathbf{5}$. The chelation sites were built first and then the 1,2,4-thiadiazole rings were introduced. Therefore, the $S$-alkylation of 1,3-benzenedimethanethiol was performed with 1,5-dichloro-3-oxapentane, which was the similar method as used to convert 3 to 4 , to prepare 6 . The formation of 6 was confirmed by its ${ }^{1} \mathrm{H}$ and ${ }^{1.3} \mathrm{C}$ NMR spectra. In 6, the $\mathrm{SH}$ of 1,3-benzenedimethanethiol was replaced by a $\mathrm{SCH}_{2} \mathrm{CH}_{2} \mathrm{OCH}_{2} \mathrm{CH}_{2} \mathrm{Cl}$ signal at $3.71,3.63$, 3.56 , and $2.56 \mathrm{ppm}$, and $70.2,69.9,43.6$, and $30.3 \mathrm{ppm}$ in the ' $\mathrm{H}$ and '"C NMR spectra, respectively.

to introduce the 1,2,4-thiadiazole rings, the S-alkylation of bis(5-amino-1,2,4-thiadiazolyl)-3,3'-disulfide (7) was performed at the 3 -position of 7 in the presence of $\mathrm{K}_{2} \mathrm{CO}_{3}$. The structure of 8 was confirmed by ${ }^{1} \mathrm{H}$ and ${ }^{1.3} \mathrm{C}$ NMR. The chlorine of 6 was replaced by a 1,2,4-thiadiazole ring signal
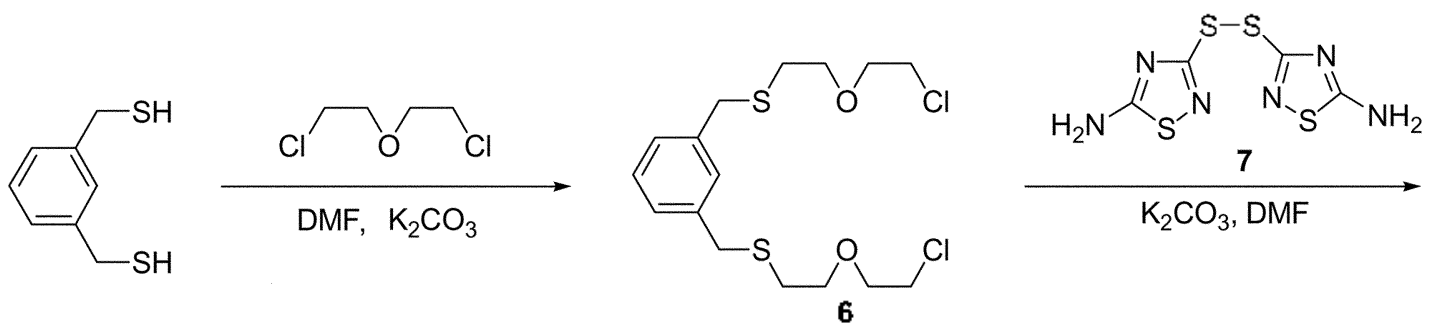

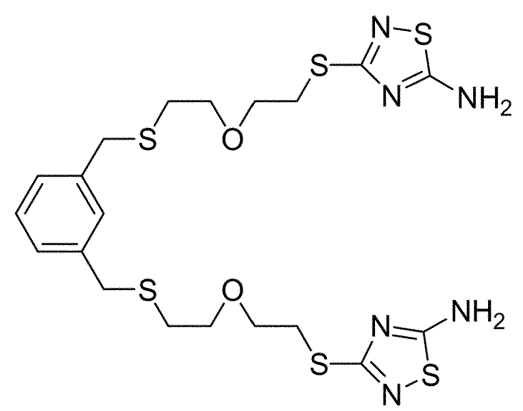

8
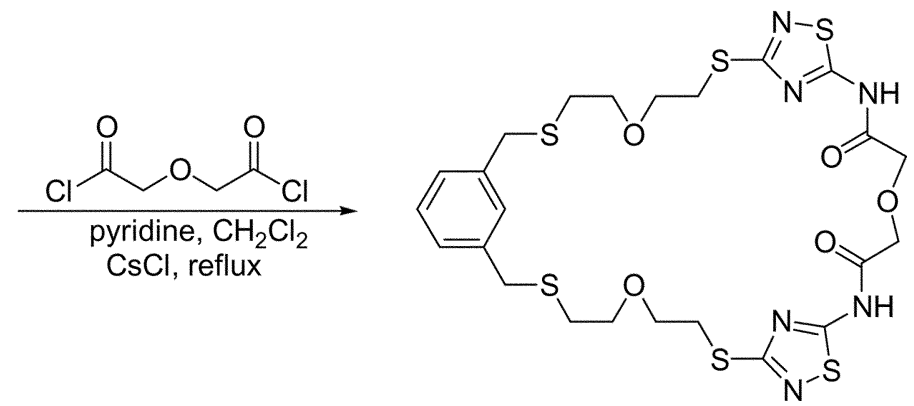

9

Scheme 2. Synthesis of macrocycle containing two 5-amino-2H-1.2.4-1hiadiasoline-3-1hione subunits. 
at $8.03 \mathrm{ppm}$ and 183.7 and $166.5 \mathrm{ppnt}$. respectively. The Salkylation was strongly supported by the typical chemical shift in which $\mathrm{CH}_{2} \mathrm{Cl}$ changes to 3.24 and $30.9 \mathrm{ppm}$ from 3.72 and $42.8 \mathrm{ppm}$ in the ${ }^{1} \mathrm{H}$ and ${ }^{13} \mathrm{C}$ NMR spectra. respectively. Target macrocycle 9 was obtained by cyclization using $N, N$-diacylation following the acylation procedure used for 4 . The structure of 9 was deternined using the same method as for $\mathbf{5}$.

\section{Experimental Section}

The IR spectra were recorded on a Jasco Report-100 spectrophotometer. The ${ }^{1} \mathrm{H}$ and ${ }^{13} \mathrm{C}$ NMR spectra were obtained using a JEOL JNM-AL 400 spectrometer at 400 $\mathrm{MHz}$ and $100 \mathrm{MHz}$ respectively with tetramethylsilane as the intental reference. NMR measurements were performed at the Central Research Facilities of Chungnan National University. Elemental analyses were carried out on an EA 1110 (CE Instrument). FAB-HRMS spectra were obtained on a JEOL-JMS HX-100/110A spectroneter at Korea Basic Science Institute, Taeduk. Taejon

The synthesis of 5-amino-2H-1,2.4-thiadiazolin-3-one $(1)^{31,32}$ and bis(5-amino-1.2,4-thiadiazolyl)-3.3'-disulfide ${ }^{33}$ followed the previous literature procedures.

5-(5-Amino-1,2,4-thiadiazol-3-yl)oxa-3-oxapentyl methanesulfonate (3). Compound (1) (5.0 g, $42.68 \mathrm{munol})$ was dissolved in heated anhydrous 1-metlyl-2-pyrrolidinone $(150 \mathrm{~mL})$ and DMF $(35 \mathrm{~mL})$ at $50{ }^{\circ} \mathrm{C}$. The clear reaction solution was cooled to room temperature, and $60 \% \mathrm{NaH}(2.6$ g. $64.00 \mathrm{mmol}$ ) was added to the above solution and the reaction mixture was stirred for $60 \mathrm{~min}$ at room temperature. The ethylene glycol dimethanesulfonate (16.8 g. 64.00 mmol) was added to the reaction mixture and heated to 55 . $60^{\circ} \mathrm{C}$ for $3 \mathrm{hr}$. The reaction mixture was cooled to roon temperature and ice water $(300 \mathrm{~mL})$ was added to the reaction mixture. The aqueous solution was extracted with chloroform $(300 \mathrm{~mL} \times 3)$. The organic solution was dried with $\mathrm{MgSO}_{4}$. The solvent was removed under reduced pressure. The residue was purified by column chromatography ( $\mathrm{SiO}_{2}$ eluent $n$-hexane : ethyl acetate $=1: 9$ ) to afford the product $(3.5 \mathrm{~g} .29 .7 \%)$

Liquid. $\mathrm{R}_{\mathrm{f}}: 0.46$ (n-hexane : ethy'l acetate $\left.=1: 9\right)$. IR $(\mathrm{KBr}$. $\mathrm{cm}^{-1}$ ): 3321, 1617, 1539. 1507, 1334, 1173. 'H NMR (400 $\mathrm{MHz} \mathrm{CDCl}_{3}, \delta$ ): 6.82 (2H. s. $\left.\mathrm{NH}_{2}\right), 4.44(2 \mathrm{H}, \mathrm{m}, \mathrm{CH}+\mathrm{O})$. $4.38\left(2 \mathrm{H}, \mathrm{m} . \mathrm{CH}_{\succ} \mathrm{OMs}\right), 3.80\left(4 \mathrm{H}, \mathrm{m} .2 \times \mathrm{CH}_{2} \mathrm{O}\right) .3 .09(3 \mathrm{H}$. s. $\left.\mathrm{CH}_{3}\right) .{ }^{13} \mathrm{C}$ NMR $\left(100 \mathrm{MHz}, \mathrm{CDCl}_{3}, \delta\right): 183.0(\mathrm{O}-\mathrm{C}=\mathrm{N})$. $166.8\left(\mathrm{H}_{2} \mathrm{~N}-\mathrm{C}=\mathrm{N}\right), 69.4\left(\mathrm{NH}_{2} \mathrm{~N}=\mathrm{COCH}_{2}\right), 69.2\left(\mathrm{MsOCH}_{2}\right)$. $68.8\left(\mathrm{MsOCH}_{2} \mathrm{CH}_{2}\right) .67 .8\left(\mathrm{NH}_{2} \mathrm{~N}=\mathrm{COCH}_{2} \mathrm{CH}_{2}\right) .37 .6\left(\mathrm{CH}_{3}\right)$. FAB-HRMS cald for $\mathrm{C}_{7} \mathrm{H}_{4} \mathrm{~N}_{3} \mathrm{O}_{5} \mathrm{~S}_{2}, 284.0375$. found 284.0378 .

1,3-Bis[5-(5-amino-1,2,4-thiadiazol-3-y])oxa-3-oxapentylthiomethyl]benzene (4). 1,3-Benzenedimethanethiol $(0.50 \mathrm{~mL}, 3.38 \mathrm{mmol})$ was dissolved in a freshly prepared 2propanol solution $(70 \mathrm{~mL}$ ) of sodium 2-propanoxide $(0.59 \mathrm{~g}$. $7.13 \mathrm{mmol}$ ). Compound (3) (0.30 g. $6.81 \mathrm{mmol}$ ) was added to the above solution and the reaction mixture was heated at reflux over 3 lur. After cooling the reaction mixture at room temperature. solvent was removed under reduced pressure.
The residue was purified by cluromatograplyy ( $\mathrm{SiO}_{2}$ : eluent $\left.\mathrm{CHCl}_{2}: \mathrm{MeOH}=15: 1\right)$ to afford product $(0.30 \mathrm{~g}, 16.3 \%)$

Liquid, $\mathrm{R}_{6}: 0.10\left(\mathrm{CHCl}_{3}: \mathrm{MeOH}=15: \mathrm{l}\right)$. IR $\left(\mathrm{KBr}, \mathrm{cm}^{-1}\right)$ : 3400. 3018, 1506, 1336. 1215. 1052. 1027. ${ }^{1} \mathrm{H}$ NMR (400 $\left.\mathrm{MHz}, \mathrm{CDCl}_{2}, \delta\right): 7.27-7.17\left(4 \mathrm{H}\right.$, m. $\left.\mathrm{C}_{6} \mathrm{H}_{4}\right) .6 .64(4 \mathrm{H}$. br, $\left.2 \mathrm{NH}_{2}\right) .4 .43(4 \mathrm{H}$. m. $2 \mathrm{C} \underline{\mathrm{H}} \mathrm{OHet}), 3.74(8 \mathrm{H}$. m. $2 \mathrm{C} \underline{\mathrm{H}}+$ $\left.2 \mathrm{CH}_{2} \mathrm{C}_{6} \mathrm{H}_{4}\right), 3.62\left(4 \mathrm{H} . \mathrm{t} . J=6.4 \mathrm{l} \mathrm{Hz} .2 \mathrm{CH}_{2}\right), 2.60(4 \mathrm{H}, \mathrm{t}, J=$ $\left.6.41 \mathrm{~Hz} .2 \mathrm{CH}_{2} \mathrm{~S}\right) .{ }^{12} \mathrm{C} \mathrm{NMR}\left(100 \mathrm{MHz} . \mathrm{CDCl}_{2} . \delta\right): 183.0(\mathrm{O}-$ $\mathrm{C}=\mathrm{N}), 167.0\left(\mathrm{H}_{2} \mathrm{NC}=\mathrm{N}\right), 138.6,129.6,128.6,127.6\left(\mathrm{C}_{6} \mathrm{H}_{4}\right)$, $70.2\left(\mathrm{CH}_{2} \mathrm{OHet} 69 . \mathrm{l}\left(\mathrm{CH}_{2} \mathrm{OCH}_{2} \mathrm{CH}_{2} \mathrm{~S}\right) .68 .2\left(\mathrm{CH}_{2} \mathrm{OCH}_{2} \mathrm{CH}_{2} \mathrm{~S}\right)\right.$. $36.7\left(\mathrm{C}_{6} \mathrm{H}_{4} \mathrm{CH}_{2}\right) .30 .9\left(\mathrm{SCH}_{2}\right)$. FAB-HRMS: $\mathrm{C}_{2 j} \mathrm{H}_{29} \mathrm{O}_{4} \mathrm{~N}_{6} \mathrm{~S}_{4}$, $(\mathrm{M}+\mathrm{l}) 545.1133$. found: 545.1138

$11,14,20,23,38,39-H e x a a z a-6,9,17,25,28$-pentaloxa-3,12,22, 31-tetrathiotetracyclo- $\left[31,3,1,1,{ }^{10,13} 1,,^{21,24}\right]$-nonatriaconta$1(37), 10(11), 13(38), 21(39), 23(24), 33(34), 35(36)$-heptaene15,19-dione (5). Compound (4) (0.3 g. $0.55 \mathrm{mmol})$ was dissolved in dichloromethane $(100 \mathrm{~mL}$ ) and pyridine $(90$ $\mathrm{mL}, 1.10 \mathrm{mmol}$ ) and diglycolyl chloride $(0.094 \mathrm{~g} .0 .55$ mmol) was added to the above solution over $24 \mathrm{hr}$. The solution was heated at reflux for additional $20 \mathrm{hr}$. After cooling the reaction mixture at room temperature. ice water $(50 \mathrm{~mL})$ was added to the reaction mixture and the mixture was stirred for $30 \mathrm{~min}$. The organic layer was separated and dried with $\mathrm{MgSO}_{4}$. The solvent was removed under reduced pressure and the residue was purified by chromatography ( $\mathrm{SiO}_{3}$ : eluent $\mathrm{CHCl}_{3}: \mathrm{MeOH}=30: 1$ ) to afford colorless solid product $(0.070 \mathrm{~g}, 19.7 \%)$.

Mp: $176.5-178.0^{\circ} \mathrm{C}, \mathrm{R}_{\mathrm{f}}: 0.49\left(\mathrm{CHCl}_{3}: \mathrm{MeOH}=30: 1\right)$. IR $\left(\mathrm{KBr}, \mathrm{cm}^{-1}\right): 3435,3165,3090,2929.1701 .1579,1279$. 1120. 1086. ${ }^{1} \mathrm{H}$ NMR (400 MHz. $\mathrm{CDCl}_{3}+\mathrm{DMSO}_{-} \mathrm{d}_{6}, \delta$ ) $12.91(2 \mathrm{H}$, br, $2 \mathrm{NH}), 7.29\left(1 \mathrm{H}, \mathrm{s}, \mathrm{CH}\right.$ of $\left.\mathrm{C}_{6} \mathrm{H}_{4}\right), 7.27-7.19$ $\left(3 \mathrm{H} . \mathrm{m} .(\mathrm{CH})_{2}\right.$ of $\left.\mathrm{C}_{6} \mathrm{H}_{4}\right), 4.48\left(4 \mathrm{H} . \mathrm{m} \mathrm{CH} \underline{\mathrm{H}}_{2} \mathrm{OHet}\right), 4.4 \mathrm{l}(4 \mathrm{H}, \mathrm{s}$, $\left((\mathrm{CO}) \mathrm{CH}_{2} \mathrm{O}\right) .3 .79$ (4H. s. $\left.\mathrm{C}_{6} \mathrm{H}_{4} \mathrm{CH}_{2} \mathrm{~S}\right) .3 .77$ (4H. m, $\mathrm{CH}_{2} \mathrm{CH}_{2} \mathrm{OHet}$ ). 3.68 (4H. t. $J=6.23 \mathrm{~Hz}, \mathrm{SCH}_{2} \mathrm{CH}_{2} \mathrm{O}$ ). 2.28 $\left(4 \mathrm{H}\right.$. t. $\left.J=6.23 \mathrm{~Hz} . \mathrm{CH}_{2} \mathrm{~S}\right),{ }^{12} \mathrm{CNMR}\left(75 \mathrm{MHz} \cdot \mathrm{CDCl}_{3}+\right.$ $\left.\mathrm{DMSO} \mathrm{d}_{6} . \delta\right) 175.4(\mathrm{O}-\mathrm{C}=\mathrm{N}) .168 .7(\mathrm{C}=\mathrm{O}), 167.2(\mathrm{~S}-\mathrm{C}=\mathrm{N})$, 139.9. 129.4. 128.5. $127.9\left(\mathrm{C}_{6} \mathrm{H}_{4}\right), 71.8\left((\mathrm{C}=\mathrm{O}) \mathrm{CH}_{2} \mathrm{O}\right) 70.2$

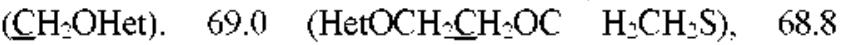
$\left(\mathrm{HetOCH}_{2} \mathrm{CH}_{2} \mathrm{OCH}_{2} \mathrm{CH}_{2} \mathrm{~S}\right), 36.7\left(\mathrm{CH}_{2} \mathrm{C}_{6} \mathrm{H}_{4}\right), 31.0\left(\mathrm{SCH}_{2}\right)$. FAB-HRMS Cald for $\mathrm{C}_{24} \mathrm{H}_{31} \mathrm{~N}_{6} \mathrm{O}_{7} \mathrm{~S}_{4}$ 643.1137. Found 643.1134 .

1,3-Bis(5-chloro-3-oxapentylthiomethyl)-benzene (6). The reaction mixture of 1.3 -benzenedimethanethiol $(20.0 \mathrm{~g}$. $0.117 \mathrm{~mol})$. 2-chloroethyl ether $(87.8 \mathrm{~g} .0 .470 \mathrm{~mol})$ and powder $\mathrm{K}_{2} \mathrm{CO}_{3}(64.8 \mathrm{~g} .0 .469 \mathrm{~mol})$ were heated to $50-55^{\circ} \mathrm{C}$ in DMF ( $150 \mathrm{~mL}$ ) over $3 \mathrm{hr}$. The solvent was removed under reduced pressure. The reaction residue was dissolved in $\mathrm{CHCl}_{3}(1000 \mathrm{~mL})$ and $\mathrm{H}_{2} \mathrm{O}(500 \mathrm{~mL})$. The organic layer was separated and washed with $\mathrm{H}_{2} \mathrm{O}(500 \mathrm{~mL})$ and dried with $\mathrm{MgSO}_{4}$ and the solvent was removed. The crude product was purified by chromatography ( $\mathrm{SiO}_{2}$ : eluent $n$-hexane ethyl acetate $=5: 1$ ) to give a colorless oil product (21.6 g. $48.0 \%$ ).

Liquid yield: (1.2 g. 55\%), $\mathrm{R}_{\text {[: }} 0.43$ (n-hexane : ethyl acetate $=4: 1)$. IR $\left(\mathrm{KBr}_{\mathrm{cm}}{ }^{-1}\right): 2956(\mathrm{CH}) .1604(\mathrm{C}=\mathrm{O}) .{ }^{\mathrm{l}} \mathrm{H}$ NMR (400 MHz. CDCl $3 . \delta): 7.30-7.01\left(4 \mathrm{H} . \mathrm{n} . \mathrm{C}_{6} \mathrm{H}_{4}\right) .3 .78$ $\left(4 \mathrm{H}\right.$. s. $\left.2 \mathrm{C}_{6} \mathrm{H}_{4} \mathrm{C}_{\underline{2}} \mathrm{~S}\right) .3 .71\left(4 \mathrm{H}, \mathrm{t} . J=5.50 \mathrm{~Hz}, 2 \mathrm{CH}_{2} \mathrm{Cl}\right), 3.63$ 
$\left(4 \mathrm{H}, \mathrm{t}, J=5.50 \mathrm{~Hz}, 2 \mathrm{CH}, \mathrm{CH}_{2} \mathrm{Cl}\right), 3.56(4 \mathrm{H} . \mathrm{t} . J=6.60 \mathrm{~Hz}$. $\left.\mathrm{SCH}_{2} \mathrm{CH}_{2} \mathrm{O}\right) .2 .56\left(4 \mathrm{H} . \mathrm{t}, J=6.60 \mathrm{~Hz}, 2 \mathrm{C}_{6} \mathrm{H}_{4} \mathrm{CH}_{2} \mathrm{SCH}_{2}\right) .{ }^{13} \mathrm{C}$ NMR (100 $\mathrm{MHz}, \mathrm{CDCl}_{\text {j. }}$ б): 138.8, 129.4. 128.4, 127.5 $\left(\mathrm{C}_{6} \mathrm{H}_{4}\right) .71 .2 .69 .9\left(\mathrm{CH}_{2} \mathrm{OCH}_{2}\right) .43 .6\left(\mathrm{CH}_{2} \mathrm{Cl}\right) .35 .3\left(\mathrm{C}_{6} \mathrm{H}_{4} \mathrm{CH}_{2} \mathrm{~S}\right)$. $30.0\left(\mathrm{C}_{6} \mathrm{H}_{4} \mathrm{CH}_{2} \mathrm{SCH}_{2}\right)$. Anal. Calcd for $\mathrm{C}_{16} \mathrm{H}_{34} \mathrm{Cl}_{2} \mathrm{O}_{2} \mathrm{~S}_{2}: \mathrm{C}$ 50.70: H 6.31; S 16.73. Found: C 50.75; H 6.34; S 17.08.

1,3-Bis \{[5-(5-amino-1,2,-thiadiazol-3-yl)thio]-3-oxapentylthiomethyl;-benzene (8). To a suspension of $\mathrm{K}_{3} \mathrm{CO}_{3}(3.27$ g. $23.66 \mathrm{~nm}$ ol) in anhydrous DMF $(60 \mathrm{~mL})$, were added compound (6) (2.26 g. $5.89 \mathrm{mmol})$ and compound (7) (7. 3.21 g. $12.14 \mathrm{munol})$ in DMF $(10 \mathrm{~mL})$ over $10 \mathrm{~min}$. The reaction mixture was heated at $50-55^{\circ} \mathrm{C}$ for $5 \mathrm{hr}$ and then heated at $70^{\circ} \mathrm{C}$ for 15 hrs. The solvent was removed under reduced pressure and the residue was dissolved in $\mathrm{H}_{3} \mathrm{O}$ (50 $\mathrm{mL}$ ) and ethyl acetate $(200 \mathrm{~mL})$. The organic layer was separated and dried with anhydrous $\mathrm{MgSO}_{4}$. Solvent was removed under reduced pressure to afford product. The crude product was purified by chromatography ( $\mathrm{SiO}_{2}$ : eluent chloroform : methanol $=20: 1)$ to give oil product $(1.18 \mathrm{~g}, 34.7 \%)$.

$\mathrm{R}_{\mathrm{f}}: 0.13\left(\mathrm{CHCl}_{3}: \mathrm{MeOH}=15: 1\right)$. IR $\left(\mathrm{KBr}, \mathrm{cm}^{-1}\right): 3300$ $3141,2918,1610,1515 .{ }^{1} \mathrm{H}$ NMR (DMSO-d $6,400 \mathrm{MHz} . \delta$ ): $8.03(4 \mathrm{H} . \mathrm{s}, 2 \mathrm{NH}) .7 .17-7.27\left(4 \mathrm{H}, \mathrm{m}, \mathrm{C}_{6} \mathrm{H}_{4}\right), 3.76(4 \mathrm{H}, \mathrm{s}$. $\left.\mathrm{C}_{6} \mathrm{H}_{3} \mathrm{CH}_{2} \mathrm{~S}\right) .3 .62\left(4 \mathrm{H} . \mathrm{t} J=6.23 \mathrm{~Hz} .2 \mathrm{SCH}_{2} \mathrm{CH}_{2} \mathrm{OCCH}_{2} \mathrm{CH}_{3}\right)$, $3.53\left(4 \mathrm{H}_{3} \mathrm{t}, J=6.60 \mathrm{~Hz} . \mathrm{SCH}_{2} \mathrm{CH}_{2} \mathrm{OCH}_{2} \mathrm{CH}_{2}\right) .3 .24(4 \mathrm{H} . \mathrm{t}, J$ $\left.=6.23 \mathrm{~Hz} . \mathrm{SCH}_{2} \mathrm{CH}_{2} \mathrm{OCH}_{2} \mathrm{CH}_{2}\right), 2.55(4 \mathrm{H} . \mathrm{t}, J=6.60 \mathrm{~Hz}$. $\left.\mathrm{C}_{6} \mathrm{H}_{4} \mathrm{CH}_{3} \mathrm{SC} \underline{\mathrm{H}}_{2}\right) .{ }^{13} \mathrm{C}$ NMR (DMSO-d $\mathrm{d}_{6}, 100 \mathrm{MHz} . \delta$ ): 183.7 $(\mathrm{S}-\mathrm{C}=\mathrm{N}) .166 .5(\mathrm{~N}-\mathrm{C}=\mathrm{N}) .139 .5 .130 .1 .129 .1 .128 .1\left(\mathrm{C}_{6} \mathrm{H}_{4}\right)$, 70.5, 69.6, $\left(\mathrm{CH}_{2} \mathrm{OCH} \mathrm{H}_{2}\right), 36.0\left(\mathrm{C}_{6} \mathrm{H}_{4} \mathrm{CH}_{2} \mathrm{~S}\right) .30 .9 .\left(\mathrm{SCH}_{2} \mathrm{CH}_{3}-\right.$ $\left.\mathrm{OCH}_{2} \mathrm{CH}_{2}\right) .30 .7\left(\mathrm{C}_{6} \mathrm{H}_{4} \mathrm{CH}_{2} \mathrm{SCH}_{2}\right)$. FAB-HRMS Cald for $\mathrm{C}_{70} \mathrm{H}_{29} \mathrm{O}_{2} \mathrm{~N}_{6} \mathrm{~S}_{6 .}$ 577.0676; Found 577.0676.

$11,14,20,23,38,39$-Hexaaza-6,17,28-trioxa-3,9,12,22,25, 31-hexathiotetracyclo- $\left[31,3,1,1^{10,13}, 1,21,24\right]$-nonatriaconta$1(37), 10(11), 13(38), 21(39), 23(24), 33(34), 35(36)$-heptaene15,19 -dione (9). The synthesis of macrocycle (9) followed the same procedure of preparation of compound (5). The crude product was purified by chromatography ( $\mathrm{SiO}_{2}$ : eluent $n$-hexane : ethyl acetate $=1: 1)$ to afford product $(33.9 \%)$.

Liquid. $\mathrm{R}_{\mathrm{f}}: 0.43$ (chloroform : methanol = $\left.15: 1\right)$. IR $(\mathrm{KBr}$. $\mathrm{cm}^{-1}$ ): 3119. 2914, 1684. 1558. ${ }^{\mathrm{H}} \mathrm{H}$ NMR (400 MHz acetoned 6.8$): 7.32-7.23\left(4 \mathrm{H}, \mathrm{m}, \mathrm{C}_{6} \mathrm{H}_{4}\right), 4.63\left(4 \mathrm{H} . \mathrm{s} .2 \mathrm{COCH}_{2} \mathrm{O}\right)$. $3.82\left(4 \mathrm{H}\right.$. s. $\left.2 \mathrm{C}_{6} \mathrm{H}_{4} \mathrm{CH}_{4} \mathrm{~S}\right) .3 .71(4 \mathrm{H}$. t. $J=6.23 \mathrm{~Hz}$. $2 \mathrm{Het}-$ $\left.\mathrm{SCH}_{2} \mathrm{CH}_{2} \mathrm{O}\right) .3 .64\left(4 \mathrm{H}, \mathrm{t}=6.60 \mathrm{~Hz}, 2 \mathrm{C}_{6} \mathrm{H}_{4} \mathrm{CH}_{2} \mathrm{SCH}_{2} \mathrm{CH}_{2} \mathrm{O}\right)$. $3.39\left(4 \mathrm{H} . \mathrm{t}=6.23 \mathrm{~Hz}\right.$. $\left.2 \mathrm{HetSC} \underline{\mathrm{H}} \mathrm{CH}_{2} \mathrm{O}\right) .2 .57(4 \mathrm{H}, \mathrm{t}=6.60$ $\left.\mathrm{Hz} .2 \mathrm{C}_{6} \mathrm{H}_{4} \mathrm{CH}_{2} \mathrm{SC} \mathrm{H}_{4} \mathrm{CH}_{2} \mathrm{O}\right) .{ }^{13} \mathrm{C}$ NMR $(100 \mathrm{MHz}$, acetone$\left.\mathrm{d}_{6}, \delta\right): 175.9(\mathrm{~S}-\mathrm{C}=\mathrm{N}) .170 .6(\mathrm{C}=\mathrm{O}) .167 .3(\mathrm{~N}-\mathrm{C}=\mathrm{N}) .140 .1$. 130.6. 129.3. $128.6\left(\mathrm{C}_{6} \mathrm{H}_{4}\right) .72 .2\left((\mathrm{CO}) \mathrm{CH}_{2} \mathrm{O}\right) .71 .3,70.0$ $\left(\mathrm{CH}_{2} \mathrm{OCH}_{2}\right)$ ). $37.0\left(\mathrm{C}_{6} \mathrm{H}_{4} \mathrm{CH}_{2}\right), 32.2\left(\mathrm{HetSCH}_{2} \mathrm{CH}_{2} \mathrm{O}\right) .31 .3$ $\left(\mathrm{C}_{6} \mathrm{H}_{4} \mathrm{CH}_{2} \mathrm{SCH}_{2}\right)$. FAB-HRMS Cald for $\mathrm{C}_{34} \mathrm{H}_{31} \mathrm{O}_{5} \mathrm{~N}_{6} \mathrm{~S}_{6}$. 675.0680: Found 675.0681 .

Acknow ledgement. This work was supported by a Grant from Chungnam National University in 2003.

\section{References}

1. Kickham, J. E.; Loeb, S. J. Inorg. Chem. 1995. 34. 5656.
2. Kickham. J. E.: Loeb. S. J. Horg. Chen. 1994, 33.4351

3. Kickham. I. E.: Loeb. S. I. J. Chem. Soc. Chem. Commm. 1993. 1848.

4. Kickham. J. E.: Loeb. S. T.: Murphy. S. L. J. Am. Chem. Soc 1993. 115.7031 .

5. Shionoya, M.: Kimura. E.; Shiro. M. J. Am. Chem. Soc. 1993. 115.6730 .

6. Van Staveren. C. T.: Van Eerden. T.: Van Veggel. F. C. I. M.: Harkema. S.: Reinhoudt. D. N. J. Am. Chen. Soc. 1988. 110. 4994.

7. Reetz. M. T.: Neimever C. M:- Hermes. M.; Goddard. M. Angew. Chem. Int Ed. Engl 1992, 31, 1017.

8. Alberts, A. H. Timmer. K: Noltes. J. G.: Spek, A. L. $J$. Am. Chem. Soc. 1979.101.3375.

9. Aoyama. Y.: Yamagishi. A.: Asagawa. M.: Toi. H.: Ogoshi. H. $J$. Ant Chent Soc. 1988. 110.4076.

10. Aoyama. Y: Asakawa. M. Yamagishi, A.: Toi, H.: Ogoshi, H. J An. Chem Soc. $1990,112.3145$.

11. Mizutani. T:; Ema, T; Yoshida. T; Kuroda, Y.: Ogoshi. H. horg Chem. 1993. 32.2072.

12. Mizutani. T.: Ema. T.: Tomita. T.: Kuroda. Y.: Ogoshi. H. J. Ant Chent. Soc. 1994. 116.4240.

13. Corradini, R.: Dossena. A.: Impellizzer, G.: Maccartone, G.; Marchelli, R.: Rizzarelli, E.: Sartor, G.: Vecchio. G. J. Am. Chem. Soc. $1994,116,10267$

14. Kuroda. Y.: Kato. Y.: Higashioji. T.: Hasegawa. T.: Kawanami. S.: Takahashi. M.: Shiraishi. N.: Tanabe. K.: Ogoshi. H. J. Am. Chen. Soc. 1995. 117. 10950.

15. Zheng. J.-Y.: Konishi. K; Aida, T. Terahedron 1997, 53.9115

16. Mizutani. T.: Yagi. S.: Honmaru. A.: Murakami. S.: Furusyo, M. Takagishi. T.; Ogoshi. H. J. Org. Chem. 1998. 63,8769.

17. Clark. T. L.: Stezowski. J. J. Am. Chem. Soc. 2001. 123.9880

18. Clark. I. L:: Booth. B. R.: Stezowski. J. T. J. Ant Chem. Soc. 2001. 123.9889 .

19. Brown.. S. E.; Haskard. C. A.; Easton. C. J.; Lincoln. S. F. J Chem. Soc. Faraday Trans. 1995, 91, 1013

20. Crumbliss. A. L.: Batinic-Haberle. I.; Spasojevic, I. Pure \& Appl. Chem. 1996. 68. 1225.

21. Cameron. B. R.: Loeb. S. T.: Yap. G. P. A. horg Chen 1997. 36. 5498.

22. Furusho. Y: Kimura. T.: Mizuno. Y.: Aida, T. J. Am. Chem. Soc. 1997. $119,5267$.

23. Murphy, S. L.: Loeb, S. J.: Shimizu. G. K. H. Tetrahedron 1998. 54.15137.

24. Sugasaki. A.: Ikeda. M.: Takeuchi. M.: Robertson1. A.: Shinkai. S J. Chem. Soc. Perkin Trans. I 1999. 3259.

25. Seneque. O.: Rager, M.-N.; Giorgi, M.: Reinaud. O. J. Ant Chent Soc. 2000, 122,6183

26. Mitoka. Y: Tsukiji. S; Hiraoka, T; Kasahi, N.: Shinkai, S: Hamachi. I. Tetahedron Lett. 2001. 42. 7059.

27. Van Manen. H.-T.: Folkens. R. H.: Nibbering. N. M. M.: Van Veggel. F. C. T. M.: Reinhoudt. D. N. J. Org Chent 2001. 66. 4643 .

28. Kubo. Y; Ohno, T: Yamanaka, J; Tokita, S.: Iida. T; Ishimaru. Y. J. Am. Chem. Soc. 2001, 123. 12700

29. Stnith. C. B.: Stephens. A. K. W.: Wallwork. K. S.: Lincolt1. F.: Taylor. M. R.: Wainwright. K. P. Inorg Chent 2002. +1 . 1093.

30. Chiba. J.: Tanaka, K.: Ohshiro. Y: Miyake, R.: Hiraoka. S.; Shiro, M.; Shionova, M. J. Org. Chem 2003, 68,331

31. Cho. N. S: Ra, C. S.: Ra. D. Y; Song. J. S.; Kang, S. K. J. Heterocyclic Chem. 1996. 33. 1201.

32. Ra. D. Y: Cho. N. S.: Moon. J. H.: Kang. S. K. J. Heterocychic Chent 1998. 35. 1435.

33. Cho. N. S.: Kim. Y. H.: Park, M. S.; Kim, E. H.; Kang, S. K.: Park. C. Heterocycles $2003,60,1401$ 